\title{
A practical Shunt Capacitor Placement Algorithm in Distribution Network
}

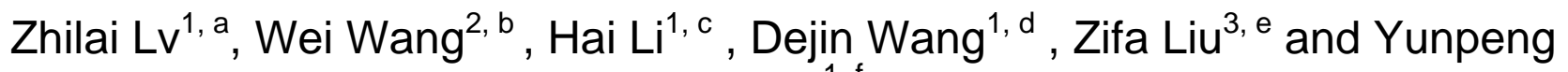 \\ Wang $^{1, f}$ \\ ${ }^{1}$ Beijing XUJI Electric Co.,Ltd., Beijing 100085, China; \\ ${ }^{2}$ School of Machine and Electric Engineering, Shandong Science and Technology University, Taian \\ 271019, China; \\ ${ }^{3}$ School of Electrical and Electronics Engineering, North China Electric Power University, Changping \\ District, Beijing 102206 \\ ahelen_Ir@163.com, 'bangwei-dongfang@163.com 'lihai@bjxj-xjgc.com, \\ dwangdejin23@sina.cn, ${ }^{\mathrm{e}} 327641896 @ q q . c o m,{ }^{f}$ hrbwang@sina.com
}

\begin{abstract}
Keywords: distribution network, capacitor shunt placement, matching power flow, sensitivity, cycle compensation method.
\end{abstract}

\begin{abstract}
For capacitor shunt placement the proposed methods do not consider the following conditions; there are few measures and the first compensation points are easily over compensation in distribution networks. So this paper proposes a practical method that can full of utilize the available measures and avoid over compensation for the first compensation point. First, the non-measured measures are evaluated by the improved matching power flow. Secondly, the step-by-step reactive power programming method based on sensitivity is used to find compensation location and capacity. Finally, the cycle compensation method is used to eliminate over compensation and better compensation results are get. The practical 85 nodes feeder verifies the accuracy and practical for the proposed algorithm.
\end{abstract}

\section{Introduction}

Research on capacitor shunt placement in distribution networks is of great realistic and theoretical significance. It can reduce the line loss effectively, improve the economic benefit. The problem is a nonlinear integer programming trouble in mathematics.

If compensation point number, location, and compensation capacity are simultaneously optimized, the search space is huge and easy to fall into the curse of dimensionality in distribution networks. Therefore, the method of the present study is mainly to find location firstly, and then determine the compensation capacity. That is uses the method of sensitivity [1-2], reactive secondary precise moment [3], the power load impedance [4] and hierarchical clustering method [5] to select compensation point. Then the number of compensation groups is calculated by using the method of mathematical programming or intelligent optimization algorithm. The disadvantage of sensitivity method is easily lead to over compensation, and it is difficulty to deal with the mutual influence between the compensation locations. Reference [6] considers the influence between the compensation points by second order network loss reactive sensitivity matrix to a certain extent. But the compensation capacity and test method for choosing node size will affect the study results. This is difficult to avoid in a separate source and capacity optimization. Reactive power secondary precise moment, the power load impedance or methods of hierarchical clustering method can avoid the influence of virtual high sensitive nodes to a certain extent. But they still rely on the reactive power compensation before network tide. Dynamic impact of network tide and sensitivity after the installation of reactive power compensation capacity are not considered. The number of nodes and number of partitions are not easy to determine.

Finally, the proposed methods are usually assumed that the load is measured at the maximum, average and minimum load conditions. But the above data is difficult to be given by the distribution network company that affects the practicability of the algorithm. 
For dealing with the above problems, this paper eliminates the over compensation phenomenon and proposes a practical parallel compensation method for distribution network.

\section{Improved matching power flow}

According to Pseudo measurement power flow calculation based on non measurement load of matched power flow calculation, such as the branch power, current and node voltage value can be calculated. The smaller the error between the calculated values and the measured values, the more likely that the estimate is more close to the actual value. The objective function of the pseudo measurement estimation of the non measurement nodes with the minimum error is given as below:

$$
\begin{gathered}
\min \varepsilon=\sum_{i=1}^{n_{V}} w_{V, i}\left|V_{i, w}-V_{i}\right|+\sum_{i j=1}^{n_{I}} w_{I, i j}\left|I_{i j, w}-I_{i j}\right| \\
\quad+\sum_{i j=1}^{n_{p}} w_{p, i j}\left|P_{i j, w}-P_{i j}\right|+\sum_{i j=1}^{n_{Q}} w_{Q, i j}\left|Q_{i j, w}-Q_{i j}\right|
\end{gathered}
$$

where $w_{V, i}, w_{I, i j}, w_{P, i j}, w_{Q, i j}$ are the weight of the real-time measurement variables of the node voltage amplitude, branch current amplitude, branch active power and reactive power respectively; $n_{V}, n_{I}, n_{p}, n_{Q}$ are the numbers of nodes with real-time measurement of node voltage amplitude, branch current amplitude, active power and reactive power of the branch respectively; $V_{i, w}, V_{i}$ are the voltage amplitude flow calculation and real-time measurement of the node i respectively; $I_{i j, w}, I_{i j}$ are the branch current amplitude flow calculation value and real-time measurement value of node number i , j respectively; $P_{i j, w}, P_{i j}$ 和 $Q_{i j, w}, Q_{i j}$ are the branch active power, reactive power flow calculation and real time measurement value of the node number $i, j$ respectively.

The equation above also satisfies the power flow, branch current and node voltage constraints.

The flow algorithm of improved matching algorithm is presented:

1、 Initialization, the admittance matrix is formed according to the network topology structure;

2、 Calculate the load pseudo measurement by using the first - end coefficient method, and calculate the distribution coefficient of the load power mismatch $\boldsymbol{\alpha}, \boldsymbol{\beta}$;

3、 The root node of the average voltage as the node voltage, the amount of power mismatch $\Delta P_{\Sigma}, \Delta Q_{\Sigma}=0$; the number of iterations $k=1$;

4、 Regard $P_{D i}^{m}+\alpha_{i} \Delta P_{\Sigma}$ and $Q_{D i}^{m}+\alpha_{i} Q P_{\Sigma}$ as the new load pseudo measurement data;

5. The voltage of each node is calculated by a power flow calculation;

6、 The power loss of the root node is $\Delta P_{\Sigma}, \Delta Q_{\Sigma}$ according to the node voltage;

7、 Determine whether the value of the target function type (1) is less than the given value, if it is less than the given value, go to step 8 , otherwise, go to step 4

8、 Calculate the pseudo measurement value of the non real time measurement load.

Through the above algorithm, we can make full use of all kinds of real time measurement. The algorithm can improve the accuracy of the estimation of the non measurement load.

\section{Objective function for shunt capacitor placement}

This paper takes the maximum ratio of investment and benefit as the target.

$$
\operatorname{Max} F=\frac{\left(\Delta P_{C}^{\max } \times t_{\max }+\Delta P_{C}^{\text {normal }} \times t_{\text {normal }}+\Delta P_{C}^{\min } \times t_{\min }\right) \times K_{D}}{\sum_{j=1}^{N_{c}}\left(Q_{c j} \times C_{c j} \times K_{c j}\right) \times \frac{\gamma \times(1+\gamma)^{n}}{(1+\gamma)^{n}-1}+N_{c} \times C_{o p} \times K_{c j}}
$$

Where $\Delta P_{c}^{\max }, \Delta P_{c}^{\text {normal }}, \Delta P_{c}^{\min }$ are saving the loss in the largest, the general, the minimum load condition respectively, $t_{\max }, t_{\text {normal }}, t_{\min }$ are the year running time in the largest, the general, the minimum load condition respectively; $K_{D}$ is the electricity price; $N_{c}$ is the group number of compensation capacitor; $Q_{c j}$ is the capacity of capacitor; $C_{c j}$ is the single group capacity cost for compensating 
capacitor; $K_{c j}$ is the compensation group number; $\gamma$ is the discount rate; $n$ is the operation time of capacitor; $C_{o p}$ is the operation cost of single group capacitor.

The equation above also satisfies the power flow, branch current and node voltage constraints.

\section{Sensitivity method}

The following method of sensitivity only considers the relationship between the reactive component of the current and the loss of the network.

$$
\Delta P_{\text {iopt }}=2 \sum_{j=1}^{b} t_{i j} I_{j} R_{j}-\frac{\left(\sum_{j=1}^{b} t_{i j} I_{j} R_{j}\right)^{2}}{\sum_{j=1}^{b} t_{i j} R_{j}}
$$

Where $I_{j}$ is the reactive component of the current before is incorporated into the branch $j$;if the branch $\mathrm{j}$ is on the path of the node $\mathrm{i}$ to the root node, $t_{i j}= \pm 1$; if the branch $\mathrm{j}$ is not on the path of the node $\mathrm{i}$ to the root node, $t_{i j}=0$; If the direction of the road $\mathrm{j}$ is the same as the road, $t_{i j}=1$,else $t_{i j}=-1$; $R_{j}$ is the resistance of the branch $\mathrm{j} ; b$ is the branch number.

According to the formula (3), the changes of the active power loss after the parallel units' reactive power can be calculated quickly. Then it can fast calculate the largest sensitivity of nodes.

\section{Loop compensation method for shunt capacitor placement}

In order to get a greater investment returns ratio, the specific algorithm flow is shown in Figure 1.

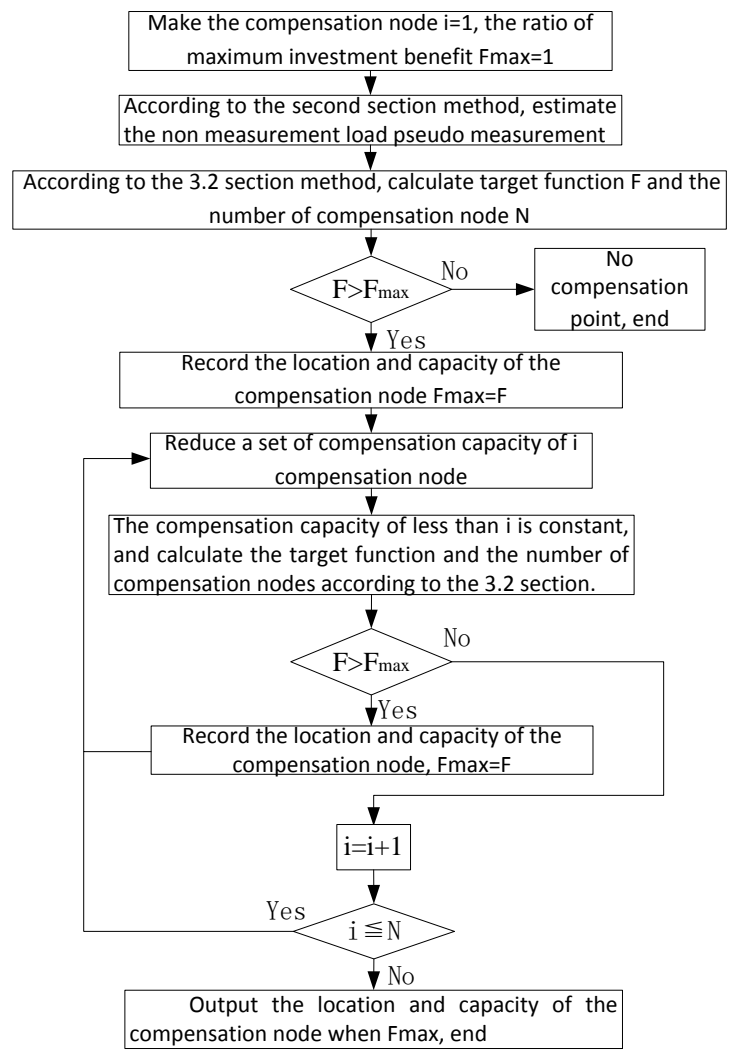

Fig.1 Flow char of algorithm 


\section{Examples and analysis}

As is shown in Figure 2, the feeder has 85 nodes, root node voltage is $10 \mathrm{kv}$. Active power supply of a year is $20700000 \mathrm{kwh}$. Maximum active load of a year is $5800 \mathrm{kw}$. The current of the feeder load 7 is $2.63 \mathrm{kA}$. The current of the feed line 15 is $1.93 \mathrm{kA}$ under the maximum load condition. Feeder line model is LGJ-120. The planning period is 5 years. The price is $0.52 ¥$ / degree. The discount rate is 0.1. Parameters of the system can be find in reference[7].

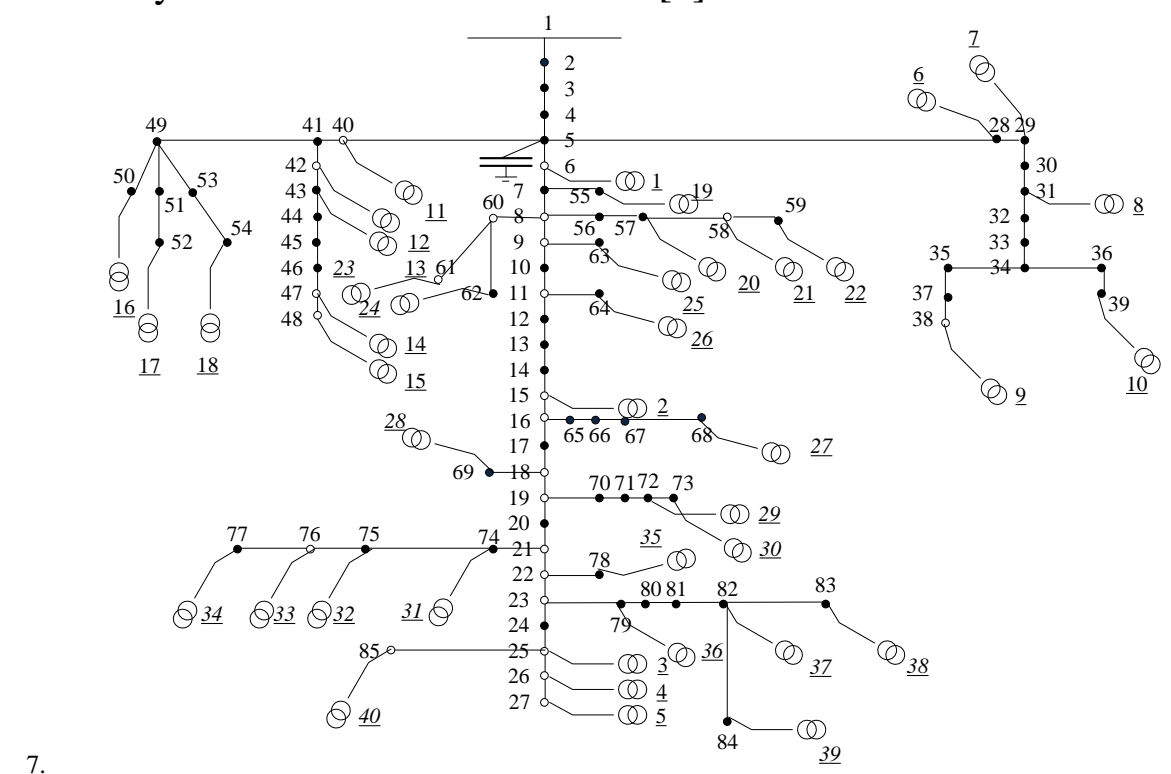

Fig.2 85 nodes practical feeder

The improved matching power flow results are shown in Table 1.

Tab.1 Non-measured measures estimated values(active power unit kw, reactive power unit kvar)

\begin{tabular}{ccccccccc}
\hline $\begin{array}{c}\text { Load } \\
\text { number }\end{array}$ & $\begin{array}{c}\text { active } \\
\text { power }\end{array}$ & $\begin{array}{c}\text { reactive } \\
\text { power }\end{array}$ & $\begin{array}{c}\text { Load } \\
\text { number }\end{array}$ & $\begin{array}{c}\text { active } \\
\text { power }\end{array}$ & $\begin{array}{c}\text { reactive } \\
\text { power }\end{array}$ & $\begin{array}{c}\text { Load } \\
\text { number }\end{array}$ & $\begin{array}{c}\text { active } \\
\text { power }\end{array}$ & $\begin{array}{c}\text { reactive } \\
\text { power }\end{array}$ \\
\hline 1 & 160.3 & 108.1 & 16 & 79.7 & 55.1 & 31 & 39.3 & 28.2 \\
2 & 79.6 & 55.8 & 17 & 16.7 & 10.8 & 32 & 25.2 & 15.6 \\
3 & 79.8 & 55.4 & 18 & 165.7 & 117.3 & 33 & 17.1 & 11.2 \\
4 & 162.9 & 110.3 & 19 & 80.8 & 55.4 & 34 & 81.2 & 55.7 \\
5 & 80.1 & 56.5 & 20 & 161.4 & 119 & 35 & 129.7 & 88.4 \\
6 & 24.5 & 17.9 & 21 & 65.8 & 44.2 & 36 & 61.8 & 43.6 \\
7 & 165.4 & 114.4 & 22 & 41.7 & 28.1 & 37 & 164.3 & 108.8 \\
8 & 39.7 & 28.1 & 23 & 24.3 & 16.1 & 38 & 158.9 & 105.6 \\
9 & 411.2 & 282.2 & 24 & 128.6 & 88.8 & 39 & 15.9 & 10.6 \\
10 & 160.9 & 109.8 & 25 & 63.4 & 42.9 & 40 & 133.9 & 86.5 \\
11 & 160.4 & 116.3 & 26 & 161.2 & 113.2 & 41 & 129.9 & 95.4 \\
12 & 81.5 & 54.8 & 27 & 24.7 & 16.5 & 42 & 162.6 & 106.3 \\
13 & 260.4 & 174.6 & 28 & 81.2 & 56.4 & 43 & 158.6 & 111.5 \\
14 & 169.0 & 105.9 & 29 & 77.4 & 58.7 & 44 & 65.3 & 43 \\
15 & 1019 & 691.8 & 30 & 132.7 & 89.6 & 45 & 83.2 & 59 \\
\hline
\end{tabular}

According to Table 1, the pseudo measurement are shown in Table 2.

Tab.2 Results of var optimal programming

\begin{tabular}{ccc}
\hline Compensation method & location & Compensation capacity (kvar) \\
\hline The method of this paper & Node 71,79 & 600,600 \\
Sensitivity method & Node 71,80 & 1000,150 \\
\hline
\end{tabular}

From table 2, it can be concluded that the sensitivity method is easy to cause first compensation node over compensation. 
Table 3 gives the comparison of the main economic and technical indexes of the method of this paper and the method of sensitivity.

Tab.3 Index of economic and technology

\begin{tabular}{cccc}
\hline & Investment benefit ratio & Line loss rate & the lowest node voltage $(\mathrm{kV})$ \\
\hline The method of this paper & 2 & $3.91 \%$ & 9.64 \\
Sensitivity method & 1.93 & $3.95 \%$ & 9.57 \\
\hline
\end{tabular}

From table 3, it can see that this method can be improved both in economy and technology compared with the sensitivity method in reactive power compensation.

\section{Summary}

Parallel compensation method for capacitor in distribution network is presented in this paper. Example results show that the proposed algorithm improves the economic and technical indicators of reactive power compensation. The algorithm has strong practicability and accuracy. The algorithm is entirely easy to develop practical reactive power optimization of distribution network planning software. The method can increase more the utility's economic benefit than the exciting sensitivity method.

\section{References}

[1]. Baran M E, Wu F F. Optimal capacitor placement on radial distribution systems[J]. IEEE Trans. Power Delivery, 1989, 4(1): 735-734.

[2]. Baran M E, Wu F F. Optimal sizing of capacitors placed on radial distribution systems[J]. IEEE Trans. Power Delivery, 1989, 4(1): 734-743.

[3]. Zhang Tingchang, Geng Guangfei. Reactive power optimization for medium voltage distribution network based on improved particle swarm optimization. Power System Technology 2012, 36(12): 158-162(in Chinese).

[4]. Jiao Zhihong-Cai, Zhong qin, Guo Zhizhong. Accurate moment method for optimization of capacitors in radial distribution systems[J]. Relay, 2002, 30(9): 11-14(in Chinese).

[5]. Yan Wei, Xu Zheng. Load power impedance moment method for optimal location of reactive power compensation on $10 \mathrm{kV}$ Feeder[J]. Proceedings of the Chinese Society of Universities for Electric PowerSystem andAutomation, 2005, 17(5):29-33(in Chinese).

[6]. Wang Shao, Zhou Xin. Optimization of reactive power in distribution network with hierarchical clustering and ant colony algorithm. Power System Technology,2011, 35(8):161-168(in Chinese).

[7]. Zhu Jingmin. Study on distribution network reactive power optimization allocation. Master. Shandong Science and Technology University. Chian, 2015.43-45 . (in Chinese). 